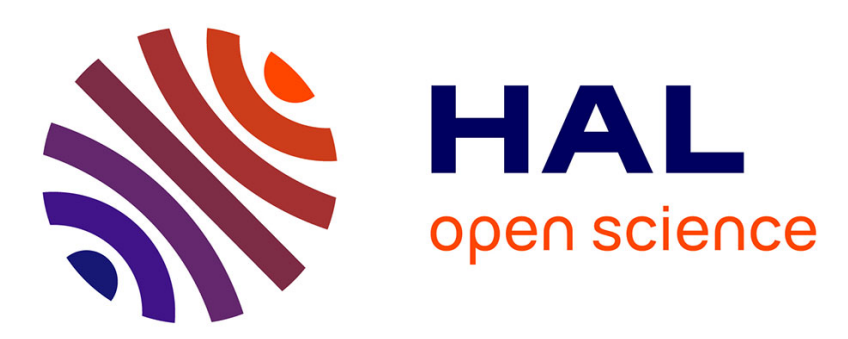

\title{
A displacive periodic antiphase model for one-dimensional platinum chain compounds
}

A. Bertinotti, C. Carcaillet, G. Jéhanno, R. Saint-James

\section{To cite this version:}

A. Bertinotti, C. Carcaillet, G. Jéhanno, R. Saint-James. A displacive periodic antiphase model for one-dimensional platinum chain compounds. Journal de Physique Lettres, 1978, 39 (3), pp.37-41. 10.1051/jphyslet:0197800390303700 . jpa-00231437

\section{HAL Id: jpa-00231437 https://hal.science/jpa-00231437}

Submitted on 1 Jan 1978

HAL is a multi-disciplinary open access archive for the deposit and dissemination of scientific research documents, whether they are published or not. The documents may come from teaching and research institutions in France or abroad, or from public or private research centers.
L'archive ouverte pluridisciplinaire HAL, est destinée au dépôt et à la diffusion de documents scientifiques de niveau recherche, publiés ou non, émanant des établissements d'enseignement et de recherche français ou étrangers, des laboratoires publics ou privés. 


\title{
A DISPLACIVE PERIODIC ANTIPHASE MODEL FOR ONE-DIMENSIONAL PLATINUM CHAIN COMPOUNDS
}

\author{
A. BERTINOTTI, C. CARCAILLET, G. JÉHANNO and R. SAINT-JAMES \\ DPh-G/PSRM, C.E.N. Saclay, Boîte Postale No 2, 91190 Gif sur Yvette, France
}

(Reçu le 28 octobre 1977, accepté le 20 décembre 1977)

\begin{abstract}
Résumé. - A partir d'une étude faite à la température ambiante nous proposons un modèle d'antiphase périodique displacive pour le composé unidimensionnel $\mathrm{CO}_{\text {def }}\left[\mathrm{Pt}\left(\mathrm{C}_{2} \mathrm{O}_{4}\right)_{2}\right] \cdot x \mathrm{H}_{2} \mathrm{O}$. $\mathrm{Ce}$ modèle, qui relie de manière simple les divers satellites discrets, rend compte des distorsions 3-dimensionnelles présentes dans la structure indépendamment de la modulation non corrélée des chaînes de platine, laquelle se traduit par des plans de diffusion continue à la température ambiante.
\end{abstract}

Abstract. - From room temperature investigations we propose a displacive periodic antiphase model for the 1-dimensional compound $\mathrm{CO}_{\text {def }}\left[\mathrm{Pt}\left(\mathrm{C}_{2} \mathrm{O}_{4}\right)_{2}\right] \cdot x \mathrm{H}_{2} \mathrm{O}$. This model, which interrelates in a simple way the various discrete satellites, accounts for the 3-dimensional distortions present in the structure independently of the uncorrelated modulation of the $\mathrm{Pt}$ chains evidenced by diffuse planes at room temperature.

The problem of the 1-dimensional compounds is dominated by the nature of the various temperature dependent displacive changes and their interrelations. In the case of the chain compounds of the platinum type the question was best formulated by Krogmann [1] who asked, some time ago, whether the distortions which are present have only to do with the metal lattice, or with that of the ligands, the cations or any other lattice atoms, too; he added further if all the other atoms were to participate in the distortions a strongly coupled static superstructure should result, which might remove any disorder phenomena.

Some evidence was given earlier, in the case of the triclinic form $\mathrm{K}_{1.62}\left[\mathrm{Pt}\left(\mathrm{C}_{2} \mathrm{O}_{4}\right)_{2}\right] \cdot 2 \mathrm{H}_{2} \mathrm{O}$, showing diffuse planes and sharp discrete satellites on the $\mathrm{X}$-rays diagrams, that a non correlated modulation of the $\mathrm{Pt}$ chains and a 3-dimensional superstructure may coexist in the same crystal lattice [2].

We present here results obtained on a dioxalato-platinate of cobalt, very similar to the violet $\mathrm{Mg}_{0.82}\left[\mathrm{Pt}\left(\mathrm{C}_{2} \mathrm{O}_{4}\right)_{2}\right] .5 .3 \mathrm{H}_{2} \mathrm{O}$ studied by Krogmann [3], where $\mathrm{Mg}$ is replaced by $\mathrm{Co}$. These results are examined in terms of a structural antiphase model which enables the satellites to be classified and related to specific lattices. It follows from this model that the lattice of the platinum atoms no longer plays the only role in establishing the static distortions.

The orthorhombic parameters of the cobalt deficient compound, very close to those of the $\mathrm{Mg}$ compound, are $a=16.54 \AA, b=14.43 \AA, c=5.70 \AA$, instead of $a=16.56 \AA, b=14.27 \AA, c=5.70 \AA$ [3]. There are four $\left[\operatorname{Pt}\left(\mathrm{C}_{2} \mathrm{O}_{4}\right)_{2}\right]$ complexes in the unit cell but the exact proportions of $\mathrm{Co}$ and $\mathrm{H}_{2} \mathrm{O}$ are not yet known.

Three principal features are displayed by the $\mathrm{X}$-ray diagrams at room temperature (Fig. 1) and (Fig. 2).

- The usual satellite diffuse planes, typical of the platinum chain compounds are visible along the most intense layer lines, perpendicular to the $\mathbf{c}^{*}$ direction,

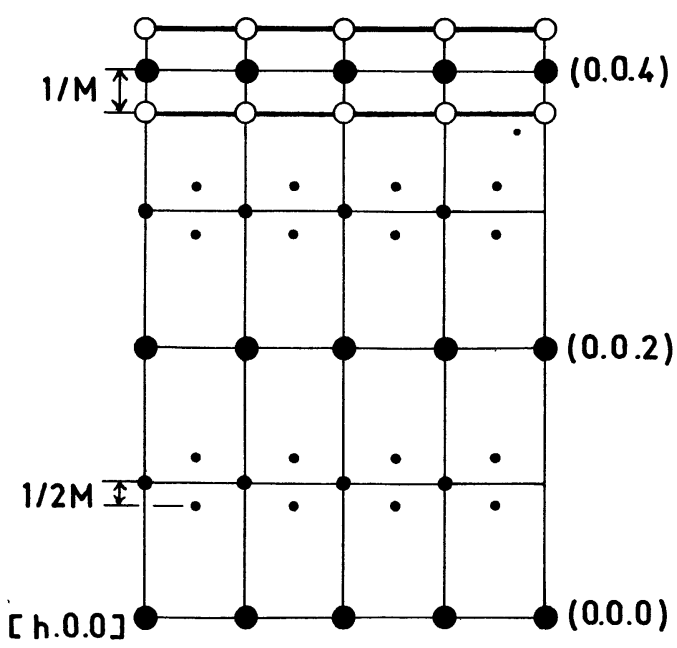

Fig. 1. - Reciprocal lattice layer hol. O- strong and weak Bragg peaks $h+k=2 n ; \bullet$ superstructure satellite ; - - - modulation satellite on diffuse satellite plane; only the visible modulation satellites (spots and planes) are indicated. 


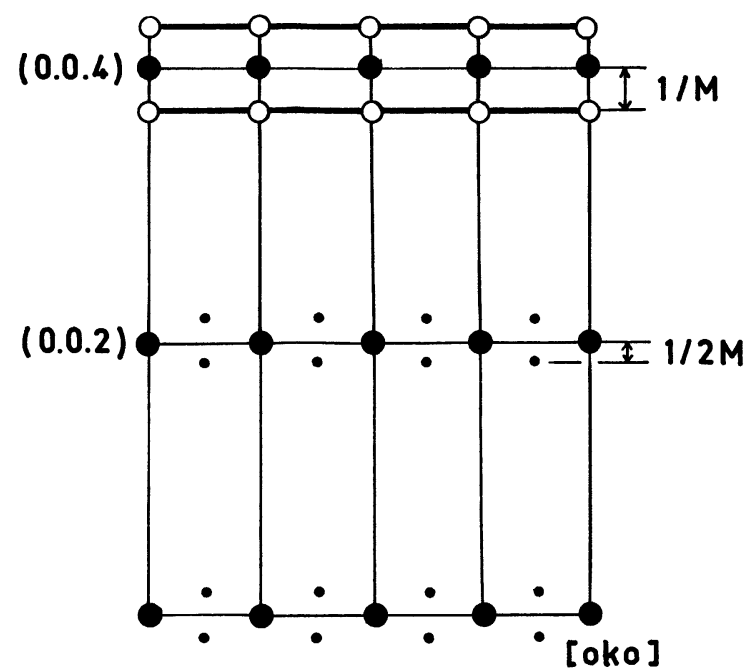

FIG. 2. - Reciprocal lattice layer okl. The symbols are the same as for figure 1 .

and corresponding to the $\mathrm{Pt}$ atoms [4]. The intensity of these planes, which are not visible in the neighbourhood of the zero layer, increases with $l$. If we take $c=5.70 \AA$ as our unit of length, they are at $Z=l \pm 1 / M$ where $l=2 n$ and $M=3.25$.

- Two types of discrete satellites are located in the $\mathbf{c}^{*}$ direction.

The most intense of these are satellites of apparently systematically absent reflections of the fundamental lattice, appearing for each level $l=n$ at $h, k, l \pm 1 / 2 M$ where $h+k=2 n+1$; we call these satellites superstructure satellites.

Another type of satellite, much weaker than the superstructure satellites at room temperature, is visible on the diffuse planes as satellites of intense reflections : we call them modulation satellites. Their distance along $\mathbf{c}^{*}$ is twice that of the superstructure satellites and their positions are at $h, k, l \pm 1 / M$, where $h+k=2 n$.

As described below both these satellites can be related in a simple way if we adopt a model of a displacive periodic antiphase structure in the crystal.

Consider a unit cell with two chains $\mathrm{A}$ and $\mathrm{B}$ of $\mathrm{Pt}$ complexes centred at $x=y=0$ and $x=y=1 / 2$, both having the same structure as that found by Krogmann [3] in the case of $\mathrm{Mg}_{0.82}\left[\mathrm{Pt}\left(\mathrm{C}_{2} \mathrm{O}_{4}\right)_{2}\right]$, but related in the unit cell by a translation which we will take provisionally equal to $(\mathbf{a}+\mathbf{b}+\mathbf{c}) / 2$. Suppose that there are antiphase frontiers perpendicular to $\mathbf{c}$ regularly spaced by a distance $M c$ Ångströms along $z$. Starting with a frontier at $z=0$, at each frontier $z=(2 p+1) M, p$ being an integer, there is an exchange of segments between the two initial chains A and $\mathrm{B}$ by a translation $(\mathbf{a}+\mathbf{b}) / 2$. The total structure is made up of supercells with a parameter $2 M$ in the c direction, each such supercell containing two coherently antiphased domains I and II. For one of these domains we introduce the following form factor

$$
f_{1}(z)=\left\{\begin{array}{l}
1 \text { for } 2 p M<z<(2 p+1) M \\
0 \text { for }(2 p-1) M<z<2 p M
\end{array}\right.
$$

and for the other $f_{2}(z)=1-f_{1}(z)$.

The Fourier transforms of these complementary functions are [5] :

$$
F_{1}(Z)=\frac{1}{2} \delta(Z)+i \varphi(Z) \quad F_{2}(Z)=\frac{1}{2} \delta(Z)-i \varphi(Z)
$$

with

$$
\varphi(Z)=\frac{1}{\pi} \sum_{m=-\infty}^{m=+\infty} \frac{1}{2 m+1} \delta\left[Z-\frac{2 m+1}{2 M}\right]
$$

- when $h+k=2 n$ the translation at the antiphase frontiers introduces a phase difference of $2 n \pi$ between the rays diffracted by each of the two domains and the two transforms must be added

$$
F_{1}(Z)+F_{2}(Z)=\delta(Z) .
$$

As a consequence the fundamental reflections are not modified

- when $h+k=2 n+1$ the phase difference is $(2 n+1) \pi$, then :

$$
F_{1}(Z)-F_{2}(Z)=\frac{2}{\pi} \sum_{m=-\infty}^{m=+\infty} \frac{1}{2 m+1} \delta\left[Z-\frac{2 m+1}{2 M}\right] .
$$

There is a splitting of the fundamental reflection into two superstructure reflections located at

$$
h, k, l \pm \frac{2 m+1}{2 M}
$$

this is the origin of the intense satellites of apparently non existent reflection, when $h+k=2 n+1$.

For the choice of the space group corresponding to the unit cell we have then to consider that $h, k, l$ with $h+k=2 n+1$ are possible reflections. The only limiting condition is observed in the $0 \mathrm{kl}$ lattice plane, where there are no fundamental (and no satellite) reflections for $l$ odd. These conditions are compatible with space group Pc2m ( No $^{\circ} 28$ in the Int. Tables for $\mathrm{X}$-ray crystallography, with the appropriate axes change). The major features of the model at room temperature can now be formulated in accordance with the space group requirements and the X-ray diffraction pattern.

The planes of the planar complexes $\operatorname{Pt}\left(\mathrm{C}_{2} \mathrm{O}_{4}\right)_{2}$ are located in the mirror planes separated by $d=2.85 \AA$ along $\mathbf{c}$, and related by a dyad perpendicular to $\mathbf{c}$. As a result there are only two possible orientations 1 or 2 for the molecules in each column with respect to the crystallographic axes. It is now necessary to define in more detail the translations between both chains 
in the unit cell, no special translation being included in space group $\mathrm{Pc} 2 \mathrm{~m}$. At room temperature we have been led to consider a translation $\frac{\mathbf{a}+\mathbf{b}}{2}$ between the $\mathrm{Pt}$ atoms, and a translation $\frac{\mathbf{a}+(1+\eta) \mathbf{b}+\mathbf{c}}{2}$, where $\eta$ is small compared to 1 , between the surrounding ligands, instead of $\frac{\mathbf{a}+\mathbf{b}+\mathbf{c}}{2}$ as mentioned previously.

It follows, as before, that both orientations 1 and 2 are present in a given mirror plane in the unit cell.

The first translation ensures that the $\mathrm{Pt}$ atoms are not sensitive to the antiphase translation $\frac{\mathbf{a}+\mathbf{b}}{2}$; there is no contribution of the $\mathrm{Pt}$ atoms to the split superstructure reflections. In direct space this means that at room temperature there is a continuity of the metal atom chain through the domain I and II alternating along c.

The second translation has several implications. If in a unit cell we consider only the oxalate groups without the Pt atoms, their contribution to the structure factor is obviously proportional to

$$
\phi(\mathbf{S})=1+\exp 2 \pi i\left[\frac{h+(1+\eta) k+l}{2}\right] .
$$

Consider the satellites with $h+k=2 n+1$, corresponding to split $h, k, l$ reflections :

i) when $l=2 n, k=0$, then $\phi(\mathbf{S})=0$, and the resulting satellites will have zero intensity, as observed along [ $h 00]$, [ $h 02]$ etc., for $h$ odd ; this fact is a consequence of the $\frac{\mathbf{a}+\mathbf{c}}{2}$ translation component between neighbouring chains. ii) When $l=2 n, k \neq 0$, then $\phi(\mathbf{S}) \neq 0$; there will be a progressive increase of the intensities with $k$, which by splitting give rise to satellite layers with a uniform increase of the intensity with $k$, as observed for example for $h k 0^{+}$or $h k 0^{-}$. iii) When $l=2 n+1$, the $\frac{\mathbf{a}+\mathbf{c}}{2}$ component will give rise to more intense superstructure satellites, as seen on $h k 1^{+}$ and $h k 1^{-}$.

Some of these characteristics are visible in figures 1 and 2 . In direct space the small $\eta \mathbf{b} / 2$ component may be related to the fact that there is no molecular centre of symmetry for the $\left[\mathrm{Pt}\left(\mathrm{C}_{2} \mathrm{O}_{4}\right)_{2}\right]$ complex, as in fact no centre of symmetry is imposed by space group $\mathrm{Pc} 2 \mathrm{~m}$. If for purposes of visualization we suppose that the centre of gravity of a bioxalate group is shifted by a quantity $\varepsilon$ along $\mathbf{b}$ from the $\mathrm{Pt}$ atom, and if we take $\eta=4 \varepsilon$ we see that the ligand columns will oscillate by $\pm \varepsilon$ along $\mathbf{b}$ around the metal chains centred at $x=y=0$ and at $x=y=\frac{1}{2}$ when going from one domain to the other (Fig. 3).

The actual form factor for such domains along $\mathbf{c}$ is certainly not the step function as defined in [1]. The *

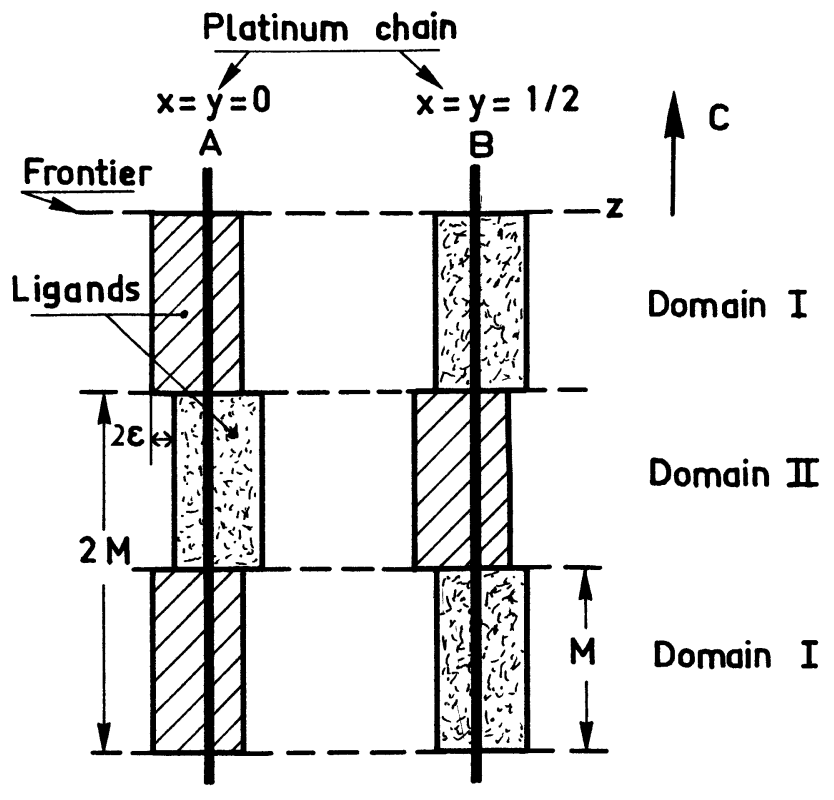

FIG. 3. - Schematic view of the periodic antiphase structure along $\mathbf{c} ; \varepsilon$ is a small quantity compared to the lattice parameters.

foregoing structure in fact implies that the period $2 M$ is an integer while the measured value is $2 M=6.5$. This fact leads us to suppose that frontiers fluctuate about mean positions, $M c$ Ångströms apart. Such a deviation from the step function causes a general weakening of the superstructure satellites and the nonappearance of the $h, k, l \pm \frac{2 m+1}{2 M}$ satellites with $m \neq 0$.

One consequence of these frontiers, which in fact correspond to structural perturbations, is that the molecules are displaced to either side, giving rise to a modulation of the atomic positions along the chain with a period $M$ half that of the supercell. This is the origin of the modulation satellites occuring at $h, k, l \pm 1 / M$, whose intensities increase with $l$. Such a modulation does not introduce additional satellites at $h, k, l \pm 1 / M$ where $h+k=2 n+1$.

Two distinct types of small atomic shifts are then occurring at the frontiers. The first type is associated with small periodic lateral shifts $\pm \varepsilon \mathbf{b}$ of the ligand columns and is related, as mentioned before, to the superstructure satellites. The second type is associated with the hindrance which occurs at the frontiers, where two successive molecules have the same orientation on one chain ; this second type is related to the modulation satellites.

Another fact suggesting the presence of a modulation of the atomic positions, other than those of the platinum atoms, is given by the intensity dissymetry observed for homologous superstructure satellites, i.e. at + or $-\frac{1}{2 M}$ for a given reflection where $h+k=2 n+1$ and $l \neq 0$.

Calculations, which have already been made for the case of periodic antiphases in alloys [6], show that if a 
modulation is present, one has to take the convolution of the Fourier transform of the form factor $f(z)$ defined above with the Fourier transform of the modulation function to obtain the intensity of the superstructure satellites, resulting in different values at + and $-\frac{1}{2 M}$. This rules out models such as the one proposed for $\mathrm{KCP}$ by Eagen et al. [7], where only a modulation of the atomic positions is present. Their model gives rise to equal values for the intensity of the homologous satellites, which led us to abandon it, though it could account for both types of satellites mentioned so far. It is only by considering the presence of both phenomena, that is periodic antiphase domains and modulation, that it becomes possible to explain the observed dissymmetry. Another model which can be ruled out is one where, besides the presence of antiphased domains, the molecules are shifted longitudinally as rigid units along $\mathbf{c}$; the dissymmetry would be always in the same sense along $\mathbf{c}^{*}$, increasing with $l$. This is not observed, but instead we observe a fairly rapid reversing of the dissymmetry when moving along a lattice row, [h01] for example.

Another important problem is the interpretation of a third type of spot not mentioned so far, which are much weaker than the superstructure satellites and very limited in number at room temperature. These faint satellites are located at a distance $\pm \frac{1}{2 M}$ along

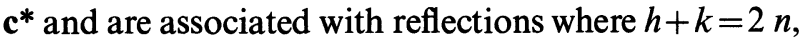
the visible satellites being connected with (2.0.1), (2.2.1), (2.4.1), (2.6.1), (1.1.1), (1.5.1)...

A tentative solution can be given by relating these satellites to the distribution of the cobalt atoms, taking account of the low temperature behaviour of the compound. By lowering the temperature slightly, besides other phenomena which are not considered here, we observe a marked increase in the number and the intensity of satellites of this type. What is noteworthy is that, among the new intense satellites, some of them appear as satellites of non visible fundamental reflections $(h 01)$, for example : $(12.0 .1),(16.0 .1)$, (18.0.1). This fact suggests that by lowering the temperature there is a contribution to the $l=1$ weak layer by atoms that have so far not been considered, and that this contribution gives rise to intense reflections split by an antiphase structure.

A simple explanation would be to assume that at a higher temperature the cobalt atoms have the distribution found by Krogmann for

$$
\mathrm{Mg}_{0.82}\left[\mathrm{Pt}\left(\mathrm{C}_{2} \mathrm{O}_{4}\right)_{2}\right] \cdot 5.3 \mathrm{H}_{2} \mathrm{O}
$$

and become ordered when the temperature is lowered, a partial order already being present at room temperature. The exact proportion of cobalt is not known but it must be not far from that of the Mg compound, the value deduced from the Kohn anomaly diffuse plane being $X=0.85$; there are less than 4 cobalt atoms per unit cell, statistically distributed at higher temperature among the 8 positions found for the $\mathrm{Mg}$ compound, i.e., on either side of the mirror plane of space group $\mathrm{Pc} 2 \mathrm{~m}$, but no longer related by the translation $\frac{\mathbf{a}+\mathbf{b}}{2}$ as in the space group Cccm. On lowering the temperature we suppose that the Co atoms have an order related to the periodic antiphase structure defined above : within $M$ unit cells along $\mathrm{c}$ the cobalt atoms would be preferentially located on one side, and for the next $M$ cells on the other side of the mirror plane. The result is that for the cobalt atoms the antiphase translation is $\frac{\mathbf{a}+\mathbf{b}+\mathbf{c}}{2}$, and no longer $\frac{\mathbf{a}+\mathbf{b}}{2}$ as for the rest of the structure. The split reflections due to the cobalt atoms are then given by the rule $h+k+l=2 n+1$, which explains for $l=1$ the satellites observed for $h+k=2 n$ mentioned before, and which may be called order satellites. Another consequence is the absence of splitting of the cobalt contribution when $h+k+l=2 n$, leaving a residual intensity at the mean lattice point in the form of a central spot, which is effectively observed even at room temperature after very long exposures, for example in the layer $l=1$ where $h+k=2 n+1$. By lowering the temperature there is an enhancement both of the central spots and of the order satellites.

For the water distribution there are no particular reasons why it should differ from that found by Krogmann for the $\mathrm{Mg}$ compound [3] except that the molecules are affected by the antiphase order.

At the present stage different points should be stressed. The model which is presented here for the cobalt platinum oxalate seems to be one of the first examples of a displacive periodic antiphase structure where the substitution concerns whole molecules. The second point we want to stress is that discrete satellite spots in the spectrum of the platinum chain compounds are not necessarily associated with a Peierls distortion. Three kinds of satellite have been distinguished : superstructure, modulation and order satellites, which coexist in our cobalt platinum oxalate with diffuse planes at room temperature; these diffuse planes correspond to an uncorrelated longitudinal modulation of the Pt atoms whereas the modulation satellites -correspond, in our interpretation, to a correlated modulation of the ligands occurring as a corollary of periodical antiphase frontiers. Similar conclusions can be drawn from the work published by Dubois [8] on violet $\mathrm{Mg}_{0.82}\left[\mathrm{Pt}\left(\mathrm{C}_{2} \mathrm{O}_{4}\right)_{2}\right] .5 .3 \mathrm{H}_{2} \mathrm{O}$. The satellites which appear in this compound on cooling may be explained, following our model, by an antiphase structure in the same way as for the cobalt compound. By lowering the temperature intense satellite spots of the superstructure type and weaker modulation satellites occur, having the periods $2 M$ and $M$ respectively. 
But unlike the present case no simple relation exists in direction and period between the modulation of the Pt atoms, evidenced by diffuse satellite planes, and the discrete modulation satellites corresponding in our interpretation to the ligands; this stresses even more the dualism of these modulations.
The last point which is noteworthy concerns the possible existence of a disorder-order transition of the cobalt cations on lowering the temperature; it can be asked if such an ordering of the non-stoichiometric ions is a general trend in the platinum chain compounds?

\section{References}

[1] Krogmann, K., in Proceedings of the German Physical Society Conference on One-Dimensional conductors, Saarbrücken, Germany, 1974, edited by H. G. Schuster (Springer, Berlin), 1975.

[2] Bertinotti, A., Bertinotti, C., Jehanno, G., C. R. Hebd. Séan. Acad. Sci. B 278 (1974) 45.

[3] Krogmann, K., Z. Anorg. Allg. Chem. 358 (1968) 97.
[4] Comès, R., Lambert, M., Launois, H., Zeller, H. R., Phys. Rev. B 8(1973) 571.

[5] Pério, P., Tournarie, M., Acta Crystallogr. 12 (1959) 1032.

[6] Jéhanno, G., Pério, P., J. Physique 25 (1964) 966.

[7] Eagen, C. F., Werner, S. A., Salllant, R. B., Phys. Rev. B 12 (1975) 2036.

[8] Dubois, J. Y., Third cycle Thesis, University of Paris-Orsay (1975). 